\title{
CODE-MIXING AND CODE-SWITCHING OF INDONESIAN CELEBRITIES: A COMPARATIVE STUDY
}

\author{
Nana Yuliana ${ }^{1}$; Amelia Rosa Luziana²; Pininto Sarwendah ${ }^{3}$ \\ 1,2,3English Literature, Faculty of Letters, Nasional University \\ Jln. Sawo Manila No. 61, Pejaten, Pasar Minggu, Jakarta Selatan 12520 \\ sylvie_surya@yahoo.com
}

\begin{abstract}
Foreign language skill presents a language variety called code-mixing and code-switching. The purpose of this study was to get some information to identify the types of code mixing and code switching frequently used by Indonesian celebrities. The study was divided into two groups. Group I was inclusive of the celebrities with native speakers parents and Group II comprised celebrities capable of speaking two or more languages. The qualitative and quantitative methods were used to analyze the code mixing and code switching with different frequency. It can be concluded that Group II use code-mixing and code-switching with a different frequency and speak foreign language more active.
\end{abstract}

Keywords: code-mixing, code-switching, Indonesian celebrities

\begin{abstract}
ABSTRAK
Kemampuan berbahasa asing menimbulkan variasi bahasa yang disebut dengan campur-kode dan alih-kode. Penelitian bertujuan untuk mendapatkan informasi guna mengetahui jenis campur-kode dan alih-kode yang dipakai oleh para selebriti Indonesia. Penelitian dibagi menjadi dua kelompok. Grup I adalah selebriti dengan orangtua penutur asli dan Grup II selebriti yang mampu berbicara dua bahasa atau lebih. Metode kualitatif dan kuantitatif digunakan untuk menganalisis campur kode dan alih kode dengan frekuensi yang berbeda. Disimpulkan, Grup II menggunakan campurkode dan alih-kode dengan frekuensi berbeda dan lebih aktif berbicara bahasa asing.
\end{abstract}

Kata kunci: campur-kode, alih-kode, selebriti Indonesia 


\section{INTRODUCTION}

Language is closely related to how people communicate with one another. Thus, in learning a language, we are also bound to people or society. In Linguistics, it is known as sociolinguistics. Sociolinguistics is the study learning about human and society. According to Trudgill (1974), sociolinguistic is part of linguistics which is concerned with language as a social and cultural phenomenon. It investigates the field of language and society that have close connections with the social sciences, especially social psychology, anthropology, human geography and sociology.

Sociolinguistics examines the interplay of language and society, with language as the starting point. Variation is the key concept, applied to language itself and to its use. The basic premise of sociolinguistics is that language is variable and changing. As a result, language is not homogeneous - not for the individual user and not within or among groups of speakers who use the same language.

The sociolinguists or people studying a language must be familiar with a code. A code is a symbol of nationalism that is used by people to speak or communicate in a particular language - a dialect, a register, an accent or a style on different occasions and for different purposes. A code is divided into code-mixing and code-switching (Stockwell, 2002). Code-mixing occurs when people mix two languages between mother tongue and English. Nababan (1993) said that code-mixing is found mainly in informal interactions. There are some reasons why people make code-mixing. Firstly, in code-mixing, bilingual speakers seem to apply some words or phrases from foreign language (pieces of one language smaller than clause), while the other language (code) functions as the base language. Secondly, bilingual speakers mix codes when there is no topic that changes, nor does the situation (Gumperz, 1982).

One of the perennial questions in bilingual research is why bilingual speakers switch from one language to another in conversational interaction. With few exception, sociolinguists who had studied code switching before the 1980s directed our attention to extra-linguistic factors such as topic, setting, relationship between participants, community norms and values, and societal, political and ideological developments. All of them were thought to influence speaker's choice of language in conversation.

Haugen (1953) stated that when the speaker of one language can produce complete meaningful utterances in the other language, it is a kind of bilingualism. However, Diebold (1964) said that a person may have no productive control over a language but be able to understand utterances in it. In such instances, linguist generally speak of "passive" or "receptive" bilingualism.

The use of code-switching and code-mixing frequently occur in conversations of bilingual speakers and they may arise at the same time but they are often used in a different context. The term code-switching is used interchangeably with code-mixing, with both terms referring to both types of language mixing. Recently, a few researchers have made finer distinctions between using code-mixing and code-switching that is to distinguish the use of two or more languages at the discourse level from switches within clauses/words (Mahootian, 2006).

The practice of alternately using two languages is called as code-switching. Code-switching is a linguistic phenomenon commonly occurring in bi- and multilingual speech communities (Mahootian, 2006). People who switch the language should have purposes, such as to quote someone, qualify message, amplify or emphasize, convey confidentiality, anger and annoyance, mark and emphasize group identity (solidarity), exclude someone from conversation, change role of speaker, rise status, add authority, show expertise and continue the last language used. (Grosjean, 1982). While code-mixing is the change of one language to another within the same utterance or in the same oral or written text (Nababan, 1993). The use of code-mixing reflects the idea that the alternation of the languages is not yet constrained (Azuma, 1998).

(Muysken, 2000) also described that code-mixing is typically divided into three main types - insertion (word or phrase), alternation (clause) and congruent lexicalization (dialect) - and the most common occurrence of codemixing variants in society is insertional code-mixing. What the writer means about insertion is insertion of material (lexical items or entire constituents) from one language into a structure of the other language. Alternation means the alternation between structures from languages. The last is congruent lexicalization of material from different lexical inventories into a shared grammatical structure: (1) Insertion: Insertion is the process of code-mixing which is conceived as something akin to borrowing: the insertion of an alien lexical or phrasal category into a given structure. (2) Alternation: It occurs between clauses meaning that alternation is used when speaker mixes his or her language with a phrase. (3) Congruent lexicalization is the influence of dialect within language use.

Meanwhile, code-switching is a phenomenon when there are two or more languages exist in a community and it makes speakers frequently switch from one language to another language (Hornberger \& McKay, 2010). According to Poplack (1980), there are three types of code-switching: (1) Intrasentential code switching; (2) Intersentential code switching; and (3) Extrasentential code switching. Intrasentential code switching occurs when the alternation of language used is below sentential boundaries.

Appel and Muysken (1987) stated that intrasentential code-switching is the alternation in a single discourse between two languages, where the switching occurs within a sentence. According to Bokamba (1988) intrasentential code-switching coincides with codemixing. Intersentential code-switching happens whereas people switching the language, Indonesian and English, between sentences or two clauses. Hughes et al., 2006 also stated that intersentential is inserting an entire phrase from the secondary language into a conversation using the other language.

Meanwhile extra sentential code-switching is a level which involves a situation in which a bilingual attaches a tag from one language to an utterance in another language such as "Darn!”, "Hey!”, "Well!”, "Look!”, etc. All types of code-switching refer to switching back and forth from one language to another to communicate to others based on the situation. So, the existence of code- 
mixing and code-switching depends on other factors that influence them to do it, for example in one situation or in different communities. Bilinguals or multilinguals have some certain reasons to mix or switch their language. Generally, the reasons include: (1) Our lizard brains take over. (2) We want to fit in. (3) We want to get something. (4) We want to say something in secret. (5) It helps us convey a thought.

Hoffmann (1991) explains several reasons why bilinguals and multilinguals switch or mix their language, it is ranging from talking about a particular topic, quoting somebody else, being emphatic about something, interjection (inserting sentence connectors), repetition used for clarification, intention of clarifying the speech content for interlocutor and expressing group entity. Saville-Troike (1986) also gives some addition to Hoffman's reasons and they are to soften and strengthen request or command, because of real lexical need and to exclude other people when a comment is intended for only a limited audience.

The aim of the research was to find out the interaction of Indonesian celebrities in using code-mixing and code-switching. This research is also expected to provide information needed by everyone studying codemixing and code-switching so they would get more understanding about types of code-mixing and codeswitching.

\section{METHODS}

Qualitative and quantitative methods were used in conducting the study by taking into account an interpretive, naturalistic approach to its subject matter and collection of a variety of empirical materials - case study, personal experience, introspective, life story, interview, observational, historical, interactional, and visual texts. Quantitative method was used to explain the phenomena by collecting numerical data that were analysed using mathematically based methods (in particular statistic). On the other hand, qualitative approach in this analysis meant the empirical research where the data were not in numbers.

There were several steps in analyzing the data. First, the data were divided into two different groups; group I consisted of celebrities whose parents were native speakers and Group II was made up of celebrities who were bilingual and multilingual. Second, the conversations were recorded and transcribed. Third, the types of code-mixing and code-switching that mostly occured in the conversations were classified. Fourth, all of the conversations using switching or mixing both in Bahasa Indonesia and English were described. Finally, each of the sentences were put into a table and the types of code-mixing and code-switching were classified and analyzed referring to Muysken (2000) and Poplack (1980) and counted them by using the theory of Butler (1985).

\section{RESULTS AND DISCUSSION}

GROUP I (Celebrities with Native Speakers Parents)
Table 1 Code-Mixing and Code Switching Group I

\begin{tabular}{|c|c|c|c|}
\hline No. & Utterances & Code-Mixing & Code-Switching \\
\hline 1 & $\begin{array}{l}\text { Nge- dance deh } \\
\text { jangan break dance. }\end{array}$ & Insertion & Intrasentential \\
\hline 2 & $\begin{array}{l}\text { Kebetulan juga lagi } \\
\text { shooting padet dan } \\
\text { butuh refreshing juga } \\
\text { tapi yang produktif. }\end{array}$ & Insertion & Intrasentential \\
\hline 3 & $\begin{array}{l}\text { Lagi kolaborasi sama } \\
\text { denim PotMixPop } \\
\text { jadi ada denim buat } \\
\text { kolaborasi di Jakarta } \\
\text { Fashion week. Emang } \\
\text { lagi iseng serius } \\
\text { merambah ke fashion. }\end{array}$ & Insertion & Intrasentential \\
\hline 4 & $\begin{array}{l}\text { Kalo diliat ada } \\
\text { details-nya, tulisannya } \\
\text { "hope love faith". }\end{array}$ & Insertion & Intrasentential \\
\hline 5 & $\begin{array}{l}\text { Iya have positive } \\
\text { energy, positive } \\
\text { feelings } d a n \ldots\end{array}$ & Alternation & Intrasentential \\
\hline 6 & $\begin{array}{l}\text {...dan juga hope-nya } \\
\text { harapannya selalu } \\
\text { ada selalu dipenuhi } \\
\text { dengan cinta. }\end{array}$ & Insertion & Intrasentential \\
\hline 7 & $\begin{array}{l}\text { I wish I was born in } \\
\text { the flower generation. }\end{array}$ & - & Intrasentential \\
\hline 8 & I have no idea. & - & Intrasentential \\
\hline 9 & $\begin{array}{l}\text { Mudah-mudahan juga } \\
\text { kalo dibilang cantik } \\
\text { ngga Cuma physically } \\
\text { semoga hatinya juga. }\end{array}$ & Insertion & Intrasentential \\
\hline
\end{tabular}

Here the celebrity mixed the language seven times and switched it nine times. Insertion was the type of code mixing mostly used. Meanwhile the type of codeswitching was intrasentential. And only one alternation in which the celebrity mixed the language with a phrase. (See Table 1)

Table 2 Code-Mixing and Code Switching Group I

\begin{tabular}{|c|c|c|c|}
\hline No. & Utterances & Code-Mixing & Code-Switching \\
\hline 1 & I am great, thank you. & - & Intersentential \\
\hline 2 & $\begin{array}{l}\text { Jadi waktu aku kecil, seki- } \\
\text { tar umur } 8 \text {, mama sempet } \\
\text { jadi host impesarial } 008 \\
\text { dan you were also there } \\
\text { where you do your magic. }\end{array}$ & Insertion & Intrasentential \\
\hline 3 & $\begin{array}{l}\text { Jadi waktu aku kecil, seki- } \\
\text { tar umur } 8, \text { mama sempet } \\
\text { jadi host impesarial } 008 \\
\text { dan you were also there } \\
\text { where you do your } \\
\text { magic. }\end{array}$ & Alternation & Intrasentential \\
\hline 4 & $\begin{array}{l}\text { Iya pindah ke LA waktu } \\
\text { aku umur 16. Di sana } \\
\text { aku high school, sempet } \\
\text { kuliah music juga and } \\
\text { then balik lagi kesini. }\end{array}$ & Insertion & Intrasentential \\
\hline 5 & It is $\mathrm{OK}$. & - & Intersentential \\
\hline
\end{tabular}


$6 \quad$ Sebenernya waktu aku di LA, mau mulai semester baru, aku dapet tawaran untuk maen java jazz dan aku merasa moment-nya lagi pas banget....

$7 \quad$... I don't know kenapa saat itu aku ngerasa it was the right thing to do dan puji tuhan...

8 pas aku balik kesini semuanya lancar dan dengan support ayah dan mama yang never ending aku jadi ya semangat berkarir di Indonesia.

$9 \quad$ Ngga sih. Emang pas aku di sana fokus aku belajar. Emang aku ngga ada pikiran untuk I am gonna try out here and Indonesia is my asset and the end of the day.

10 Iya sampai akhirnya waktu pindah ke Jakarta, mama approach aku bilang, kamu mau ngga main film? Of course. Apalagi setelah dikasih skenarionya, ceritanya aku tertarik.

11 It is a help of course.

12 It is an advantage, pasti lah. Maksudnya, aku ngga mungkin ada di sini sekarang tanpa ayah atau mama.

13 Bukan compare ya, kaya ngasih ekspektasi aja.

14 Pasti ada yang ga suka. Haters pasti ada cuma aku fokus ke berkaryanya aja sih.

15 Ayah dan mama selalu ngingetin sih focus make art, go do art.

16 Ngga jadi ceritanya tadi kan aku bilang mama mau bikin film terus mama bilang please help me nih yang jadi cowonya siapa ya and then I was looking three on personate work whatever eh .... trus ada namanya, kenapa ga ini ja Adipati Dolken?

17 Karena kebetulan di depan mata. Terus mama bilang tolong dong contact Adipati.

18 Deket. Kita sering banget manggung bareng semenjak aku balik ke Jakarta. Nih besok mau manggung lagi di-launchnya dia. Sering bikin lagu bareng.

19 I don't know.

Insertion Intrasentential

Arternation Intrasentential

Insertion Intrasentential

Alternation Intrasentential

Insertion Intrasentential

Intrasentential

Alternation Intrasentential

Insertion

Intrasentential

Insertion

Intrasentential

Alternation

Intrasentential

Alternation

Intrasentential

I think I can, karena setiap aku nyanyi penonton kayaknya enjoy.

31 I think I can, karena Insertion Intrasentential setiap aku nyanyi penonton kayaknya enjoy.

The celebrity used code-mixing twenty-five times and code-switching thirty one times but she almost used all those kinds of code-mixing and code-switching. (Table 2)

$20 \quad$ I can get kinda
emotional kalo
ngomongin soal ayah.
Cuman he is such a grea

dad.

$21 \quad$ Ini kok musiknya jadi

kayak gini si? Apa ya,

ah tuh orangnya asik,

lah both of my parent $y a$

very inspiration all.

22 Ngga cuma ayah, mama Insertion Intrasentential

jug selalu dukung,

support. Mungkin juga

yang sama film dan music

jadi makin akur lagi kaya

udah kangennya ayah

bikin album judulnya

dia rilis dalam bentuk

application dan waktu itu

pas proses bikin albumnya

ayah nanya mau duet ga?

ya mau dong.

nanti ayah kirimin

lagunya. Trus aku denger

lagunya, aku kayak

get, man

dikirim and basicly about

my relationship with him

being far away dan...

musiknya di Jakarta, aku

take vocal-nya di lemari

baju aku di $L A$.

Alternation

Intrasentential

Intrasentential

Intrasentential

Intrasentential

...cuma ada inside-inside
yang bikin kita ini lebih

Insertion

Alternation

Intrasentential

Insertion Intrasententia

Insertion Intrasentential

Intersentential 
GROUP II (Bilingual and Multilingual Celebrities)

\section{Table 3 Code-Mixing and Code Switching Group II}

\begin{tabular}{|c|c|c|c|}
\hline No. & Utterances & Code-Mixing & Code-Switching \\
\hline 1 & $\begin{array}{l}\text { Sebenernya memang } \\
\text { album ini seperti } \\
\text { warming up untuk para } \\
\text { fans, genre apa yang } \\
\text { akan aku bawa pada saat } \\
\text { di international album } \\
\text { dan liriknya seperti apa, } \\
\text { cara aku deliver the song } \\
\text { seperti apa. Dan buat } \\
\text { aku, walaupun banyak } \\
\text { orang yang berfikir wah } \\
\text { berani banget ya Agnez } \\
\text { ngeluarin album yang full } \\
\text { English and... }\end{array}$ & Insertion & Intrasentential \\
\hline 2 & $\begin{array}{l}\text { Dan buat aku, walaupun } \\
\text { banyak orang yang } \\
\text { berfikir wah berani } \\
\text { banget ya Agnez } \\
\text { ngeluarin album yang full } \\
\text { English and then I know } \\
\text { I gonna to enjoy singing } \\
\text { those songs on stage. So, } \\
\text { you know, there you go. }\end{array}$ & Alternation & Intrasentential \\
\hline 3 & $\begin{array}{l}\text { Dan buat aku, walaupun } \\
\text { banyak orang yang } \\
\text { berpikir wah berani } \\
\text { banget ya Agnez } \\
\text { ngeluarin album yang full } \\
\text { English... }\end{array}$ & Alternation & Extrasentential \\
\hline 4 & $\begin{array}{l}\text {... and then I know I } \\
\text { gonna to enjoy singing } \\
\text { those songs on stage. So, } \\
\text { you know, there you go. }\end{array}$ & - & Intrasentential \\
\hline 5 & $\begin{array}{l}\text { It's so funny cause I am } \\
\text { always been asked the } \\
\text { same question like what } \\
\text { next, what do you want in } \\
\text { your life? }\end{array}$ & - & Intrasentential \\
\hline 6 & $\begin{array}{l}\text { To be honest, aku sendiri } \\
\text { pengin jadi a better per- } \\
\text { son everyday baik secara } \\
\text { individual dan juga } \\
\text { secara musikalitas aku. }\end{array}$ & Alternation & Intrasentential \\
\hline 7 & $\begin{array}{l}\text { Sampai sekarang pun aku } \\
\text { terus ngambil kelas-kelas } \\
\text { itu. Dari kelas dance, } \\
\text { basical training, still, } \\
\text { until right now. Karena } \\
\text { buat aku, mau sebagus } \\
\text { apapun kita, itu seperti } \\
\text { skill, seperti otot yang } \\
\text { harus terus dilatih. Dan } \\
\text { memang project yang } \\
\text { kita kerjakan akan segera } \\
\text { keluar makanya sepuluh } \\
\text { hari lagi aku balik lagi ke } \\
\text { Amerika. }\end{array}$ & Insertion & Intrasentential \\
\hline 8 & $\begin{array}{l}\text { Actually my destination } \\
\text { is worldwide. }\end{array}$ & - & Extrasentential \\
\hline 9 & $\begin{array}{l}\text { Like I really want to } \\
\text { share my passion not } \\
\text { only to Indonesian, not } \\
\text { only to Asian, but basicly } \\
\text { people around the world. } \\
\text { Dan sekarang orang- } \\
\text { orang juga tau kalo USA } \\
\text { itu seperti... }\end{array}$ & Insertion & Intrasentential \\
\hline
\end{tabular}

10 Exactly, like the centre of attention baik itu dari Europe, Asia dan yang lain-lain.

11 Exactly, like the centre of attention baik itu dari Europe, Asia dan yang lain-lain.

12 Both places I would call home karena aku betah di Indonesia, aku betah di LA.

13 Ya tergantung prioritasnya aja. Karena sekarang prioritasnya sekarang lagi ada project yang mau aku luncurin, mau ngga mau aku lebih lama di sana.

14 Sebenernya bukan masalah ngajak tapi pada saat dengan timbaland, timbaland yang ngomong dia yang mau executive produserin album aku. Kita liat nanti aja gimana opportunity yang datang to our table.

15 Oke. Aku selalu bilang dream, belive and make it happen.

16 The reason why I say dream, belive, and make it happen beacause I prove it by myself. When you have a dream, and you belive in your dream, you can actually make it happen.

17 Gak ada yang bilang kalo perjalanannya itu bakal gampang tapi kamu ngga akan bisa berjalan kalau kamu ngga ada dream.

18 Kamu juga ngga akan berjalan kalau kamu ngga believe...

19 dan kamu ngga akan berjalan kalau kamu ngga try to make it happen...

20 Jadi pada akhirnya kalau Alternation Intrasentential you don't have these three you just gonna be stuck in your life, you know, forever dan ngga akan bisa maju-maju.

21 At least, the least that you can do for yourself adalah itu have a dream, believe it and try to make it happen.

Insertion Intrasentential

Alternation Intrasentential

Insertion

Intrasentential

Insertion Intrasentential

Alternation

Intrasentential

Intersentential

Intrasentential

Insertion

Intrasentential

Intrasentential

Insertion (1)

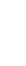

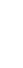

Extrasentential

The celebrity mixed the language sixteen times and switched it for twenty-one times and also used codemixing and code-switching. (Table 3 ) 
Table 4 Code-Mixing and Code Switching Group II

\begin{tabular}{|c|c|c|c|}
\hline No. & Utterances & Code-Mixing & Code-Switching \\
\hline 1 & $\begin{array}{l}\text { Saya akan mengundang } \\
\text { seorang bintang tamu, ini } \\
\text { masih muda. Kita akan } \\
\text { membahas the power of } \\
\text { woman. }\end{array}$ & Alternation & Intrasentential \\
\hline 2 & $\begin{array}{l}\text { Langsung saja, karena } \\
\text { banyak yang saya mau } \\
\text { tanya sama anak muda ini. } \\
\text { Ini dia Eva Celia. Eva, how } \\
\text { are you? }\end{array}$ & - & Intersentential \\
\hline 3 & $\begin{array}{l}\text { Nah ini ada videonya pas } \\
\text { Eva nyanyi sama Indra } \\
\text { Lesmana lewat Skype. } \\
\text { Right? Here it is }\end{array}$ & - & Intersentential \\
\hline 4 & $\begin{array}{l}\text { Aduh saya ketemu Eva } \\
\text { ini, saya merasa tua sekali } \\
\text { karena ngga tau ya. Tadi } \\
\text { di belakang saya nanya do } \\
\text { you remember me? Gitu } \\
\text { ya? }\end{array}$ & Alternation & Intrasentential \\
\hline 5 & $\begin{array}{l}\text { Ayo come come, duduk } \\
\text { sini. Saya mau nanya kamu } \\
\text { inget saya ga? }\end{array}$ & Insertion & Extrasentential \\
\hline 6 & $\begin{array}{l}\text { Iya jadi dia tuh sering } \\
\text { dibawa ibunya which is } 13 \\
\text { tahun yang lalu mungkin } \\
\text { ya? }\end{array}$ & Insertion & Intrasentential \\
\hline 7 & $\begin{array}{l}\text { Kalo ibunya nge-host kan } \\
\text { dia ga ada yang nemenin } \\
\text { ya, muter-muter keman- } \\
\text { mana tuh pernah saya } \\
\text { pangku, masih kecil segini. }\end{array}$ & Insertion & Intrasentential \\
\hline 8 & $\begin{array}{l}\text { Iya. Jadi tadi pas saya } \\
\text { ngeliat fotonya, I know } \\
\text { this girl. }\end{array}$ & Alternation & Intrasentential \\
\hline 9 & $\begin{array}{l}\text { Wow } 21 \text { tahun. Itu } 13 \text { tahun } \\
\text { yang lalu you were seven } \\
\text { or eight years old ya? }\end{array}$ & Alternation & Intrasentential \\
\hline 10 & $\begin{array}{l}\text { Apa yang saya lewatkan di } \\
\text { hidup kamu? Karena waktu } \\
\text { itu kamu and your mom } \\
\text { pindah ke luar negeri kan? }\end{array}$ & Insertion & Intrasentential \\
\hline 11 & $\begin{array}{l}\text { Jadi kamu masuk ke dunia } \\
\text { entertaint. Kamu kuliahnya } \\
\text { apa? }\end{array}$ & Insertion & Intrasentential \\
\hline 12 & $\begin{array}{l}\text { Ada yang compare ngga } \\
\text { tapi kamu? }\end{array}$ & Insertion & Intrasentential \\
\hline 13 & $\begin{array}{l}\text { Udah twenty one udah } \\
\text { punya pacar belom? }\end{array}$ & Insertion & Intrasentential \\
\hline 14 & $\begin{array}{l}\text { Sorry saya potong. Kalo } \\
\text { Eva bilang sayang papa, } \\
\text { ngga kangen tuh jauh? }\end{array}$ & Insertion & Intrasentential \\
\hline 15 & $\begin{array}{l}\text { Oooh. Your dad pasti } \\
\text { watching this right? }\end{array}$ & Alternation & Intrasentential \\
\hline 16 & Oke tell me about your dad. & - & Intrasentential \\
\hline 17 & How much you love him? & - & Intrasentential \\
\hline 18 & You love him, tell him. & - & Intrasentential \\
\hline 19 & $\begin{array}{l}\text { Oke, itu hubungan anak } \\
\text { dengan ayahnya ya. Ini } \\
\text { menarik sekali karena } \\
\text { sebenernya orangtua Eva } \\
\text { itu divorce ya? Sama. }\end{array}$ & Insertion & Intrasentential \\
\hline
\end{tabular}
wife tapi kasih sayang ke anaknya ngga akan pernah bilang ya.

21 Now, it is time for you to explain me what kind of movie?

22 Why we watch the movie?

23 Eva tadi saya nanya sama kamu kan, kalau kamu suruh pilih nyanyi or main film gimana?

24 You should think right? Karir nyanyi kamu gimana?

25 Are you a good singer? -

26 Penonton kayaknya enjoy, Insertion jadi bisa menghibur orang lah ya?

27 Untuk Adipati dan Kevin, Insertion Intrasentential saya ngga tau nih di usia kalian yang masih muda kalian berencana untuk selamanya di entertainment atau yang lainnya maybe business?

28 Sekarang lagi break? Insertion Intrasentential

29 Oke. Terima kasih buat Insertion Intrasentential semuanya yang hadir disini.

Mudah-mudahan filmnya sukses dan Eva welcome back.

30 Oke. Terima kasih buat semuanya yang hadir disini. Mudah-mudahan filmnya sukses dan Eva welcome back. How are you?

The celebrity showed that the result of mixing was twenty times and switching was thirty times. (Table 4)

The following chart (Figure 1) showed that Group II used $62 \%$ of code-switching and $38 \%$ of code-mixing. Although most of them spoke Bahasa Indonesia and English fluently, the frequency of switching the language was more than mixing. Surprisingly, the percentage of code-switching of Group I was lower than Group II, as in $53 \%$ to $62 \%$, but the percentage of code-mixing of Group I was higher than Group II, as in $47 \%$ to $38 \%$.

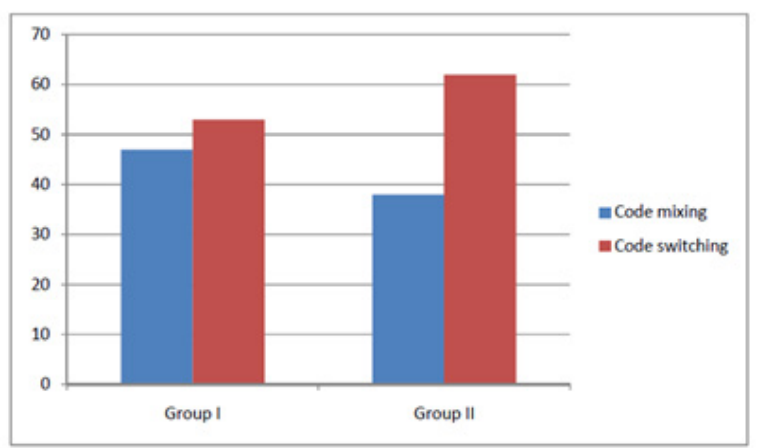

Figure 1 Code Mixing and Code Switching Charts 
The following calculation (see Figure 2 and Figure 3) showed how much alternation and insertion in codemixing and intersentential, intrasentential as well as extrasentential in switching was used:

\section{Group I:}

\section{Code mixing:}

Insertion $\quad: 6+14+6+22+7+21=76$

Alternation : $1+11+1+3+10+0=26$

Congruent : 0

Insertion

$$
\frac{76}{76+27+0} \times 100 \%=\frac{76}{103} \times 100 \%=73.7 \%=74 \%
$$

Alternation :

$$
\frac{26}{76+27+0} \times 100 \%=\frac{26}{103} \times 100 \%=25.24 \%=25 \%
$$

Congruent : 0

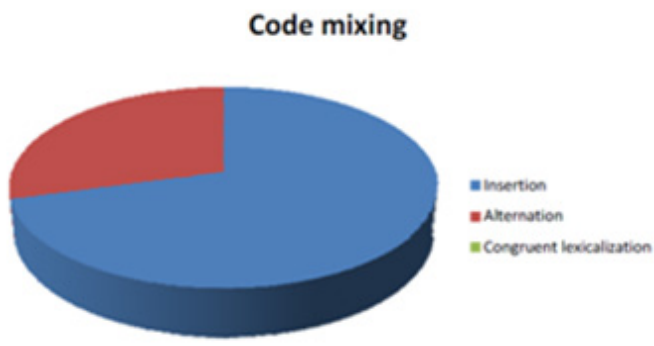

Figure 2 Code-Mixing

\section{Code switching:}

Intersentential $: 2+6+7+0+8+0=23$

Intrasentential : $25+7+25+14+21=99$

Extrasentential $: 0+0+0+0+4+0=4$

Intersentential :

$\frac{23}{23+99+4} \times 100 \%=\frac{23}{126} \times 100 \%=18.25 \%=18.2 \%$

Intrasentential :

$\frac{99}{23+99+4} \times 100 \%=\frac{99}{126} \times 100 \%=78.57 \%=78.6 \%$

Extrasentential :

$\frac{4}{23+99+4} \times 100 \%=\frac{4}{126} \times 100 \%=3.17 \%=3.2 \%$

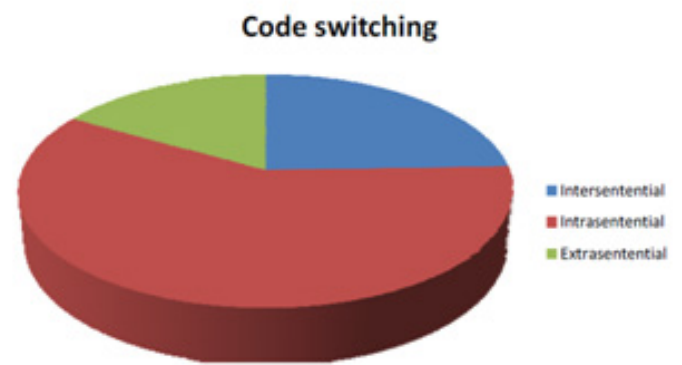

Figure 3 Code-Switching

\section{CONCLUSION}

The celebrities with native speaker parents or those who were capable of speaking more than one language fluently used code-mixing and code-switching but in different frequency. It was also proven that celebrities who were bilingual and multilingual were more active than those whose parents were from English speaking countries. Most types of code-mixing and code-switching used in Group I were insertions. On the other hand, Group II insertions were mostly used and alternations were rarely used. However, there is not any congruent lexicalization was used either in Group I or Group II. They totally did not change their accent. Code-switching that was mostly used was intrasentential and fewer extrasentential. The choice of code-mixing and code-switching of those two groups was the same but the frequency showed the difference. Both prefered using insertions in code-mixing and intrasententials in code-switching.

\section{REFERENCES}

Appel., R., \& Muysken, P. (1987). Language contact and bilingualism. London and Baltimore: Edward Arnold.

Azuma, S. (1998). Meaning and form in code-switching. In R. Jacobson (Ed.), Codeswitching Worldwide. Berlin/New York: Mouton de Gruyter.

Bokamba, E. (1988). Code-mixing and grammatical theory: a cross-linguistic study. A CrossLinguistic Study. Paper Presented at the 21st Chicago Linguistic Society Meeting, University of Chicago.

Butler, C. (1985). Statistics in Linguistics. TESOL Quarterly, 20(3), 549-552.

Diebold, A. R. (1964). Incipient bilingualism in Hymes, D. Ed. Language in Culture and Society. New York: Harper and Row.

Grosjean, F. (1982). Life with two languages. Cambridge. Cambridge: Cambridge University Press.

Gumperz, J. J. (1982). Discourse Strategies. Cambridge: Cambridge University Press.

Haugen, E. (1953). The Norwegian Language in America: A study in bilingual beaviour. Philadelphia: UP of Pennsylvania.

Hoffmann, C. (1991). An Introduction to Bilingualism. London: Longman. 
Hornberger, N. H., \& McKay, S. L. (2010). Sociolinguistics and Language Education. Great Britain: Short Run Press.

Hughes, B., Baldwin, T., Bird, S., Nicholson, J., \& MacKinlay, A. (2006). Reconsidering language identification for written language resources. In Proc. of the 5th Edition of the International Conference on Language Resources and Evaluation (LREC 2006). Genoa, Italy.

Mahootian, S. (2006). Code Switching and Mixing. In K. Brown (Ed.), Encyclopedia of Language \& Linguistics. Oxford: Elsevier.

Muysken, P. (2000). Bilingual Speech: A Typology of Code-Mixing. United Kingdom: Cambridge University Press.

Nababan, P. W. J. (1993). Sociolinguistics: Sebuah Pengantar. Jakarta: Gramedia Pustaka.

Poplack, S. (1980). Sometimes I'll start a sentence in Spanish y termino en español. Linguistics, 18, 581-618.

Saville-Troike, M. (1986). The Ethnography of Communication: An Introduction. Oxford: Basil Blackwell.

Stockwell, P. (2002). Sociolinguistics: A Resource book for students. London: Routledge.

Trudgill, P. (1974). Sociolinguistics: An introduction to language and society (Fourth). England: Penguin Books. 